\title{
NUMERICAL INVESTIGATION OF WIND INDUCED OVALLING VIBRATIONS IN SILO GROUPS
}

\author{
Jeroen Hillewaere ${ }^{1}$, Joris Degroote ${ }^{2}$, Ali Rezayat ${ }^{3}$, Steve Vanlanduit ${ }^{3}$, Geert Lombaert ${ }^{1}$, \\ Jan Vierendeels ${ }^{2}$, and Geert Degrande ${ }^{1}$ \\ ${ }^{1}$ Department of Civil Engineering, KU Leuven \\ Kasteelpark Arenberg 40, B-3001 Heverlee, Belgium \\ e-mail: \{jeroen.hillewaere,geert.lombaert,geert.degrande\}@bwk.kuleuven.be \\ ${ }^{2}$ Department of Flow, Heat and Combustion Mechanics, Ghent University \\ St. Pietersnieuwstraat 41, B-9000 Gent, Belgium \\ e-mail: \{joris.degroote,jan.vierendeels\}@ugent.be \\ ${ }^{3}$ Department of Mechanical Engineering, Vrije Universiteit Brussel \\ Pleinlaan 2, B-1050 Brussels, Belgium \\ e-mail: \{arezayat,svanlandui\}@vub.ac.be
}

Keywords: Wind-structure interaction, ovalling vibrations, wind tunnel validation, silo structure.

\begin{abstract}
Wind induced ovalling vibrations were observed during a storm in October 2002 on several empty silos of a closely spaced group consisting of 8 by 5 thin-walled silos in the port of Antwerp (Belgium). In the present study, it is investigated if the observed ovalling oscillations can be numerically predicted. To this end, the silo structures are modeled using a finite element (FE) model and the wind flow around a single silo is investigated using $3 D$ computational fluid dynamics (CFD) simulations. A wind tunnel experiment was set up to validate the CFD simulations. To explain the onset of the wind induced ovalling vibrations, the interaction of the wind field with the structural vibrations has to be taken into account. Therefore, the complex interplay of both fields is investigated with both one-way and two-way coupling simulations. It is interesting to compare the results of both coupling approaches to assess the necessity of performing the computationally much more time-consuming two-way coupling simulations. It is found in both coupling approaches that the mode shapes with the lowest natural frequencies are excited dynamically. The results in the one-way coupling simulations differ however from those in the two-way coupling, demonstrating the importance of performing fully coupled simulations for suchlike aeroelastic problems.
\end{abstract}




\section{INTRODUCTION}

Wind induced ovalling vibrations were observed during a storm in October 2002 on several empty silos of a closely spaced group consisting of 8 by 5 thin-walled silos in the port of Antwerp (Belgium) (figure 1). Numerical techniques are a valuable alternative to wind tunnel tests or full scale measurements to study the onset of these aeroelastic structural vibrations. For this purpose, the coupled wind-structure interaction (WSI) problem as a whole should be considered numerically. This implies that the 3D computational fluid dynamics (CFD) simulations of the wind flow and the structural vibrations, calculated with a finite element (FE) model should be considered as one coupled problem.

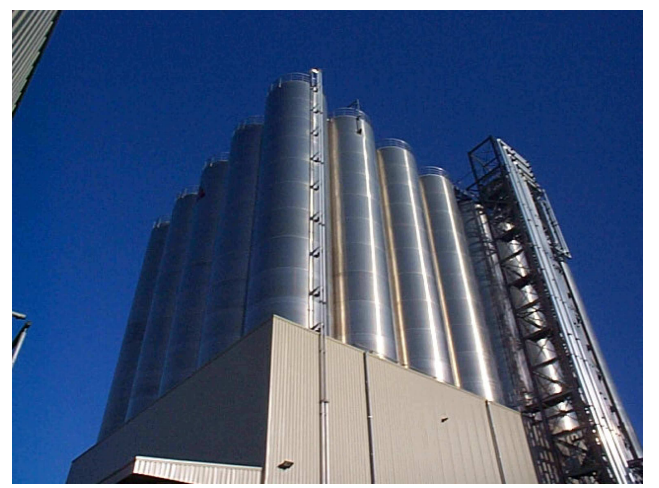

Figure 1: The 8 by 5 silo group in Antwerp.

Due to the complexity of this coupled problem when the entire 8 by 5 silo group is considered, it is advisable to first study a more simple subproblem to verify and validate the numerical procedures. Therefore, in the present paper, the case of a single 3D silo in cross flow is considered.

The outline of the paper is as follows. The dynamic properties of the silo structures will be presented first. Finite element modeling is used to determine natural frequencies and ovalling mode shapes of a silo. The 3D CFD simulation of the highly turbulent flow around a single silo is presented in the third section. In addition to the description of the applied numerical procedures and the approach to apply realistic inlet conditions a separate section is dedicated to the validation of the numerical results in a wind tunnel experiment. In the last section, the wind-structure interaction problem is considered where the numerical model for the structure and for the wind flow are coupled. The first and easiest approach investigated is the one-way coupling simulation, where the aerodynamic surface pressures on the silo surface are applied as external transient loads on the FE model of the structure. Finally, a two-way coupled simulation is performed where feedback is given in each time step from the structure to the flow field. It takes approximately five times longer to compute a single time step in the two-way coupling simulations when compared to the one-way coupling.The results of both coupling simulations are compared to assess the necessity of performing the computationally much more time-consuming two-way coupling simulations to investigate such aeroelastic phenomenon.

\section{STRUCTURAL MODEL}

Ovalling deformations of a thin-walled shell structure are defined as a deformation of the cross section of the structure without bending deformation with respect to the longitudinal axis of symmetry [11]. The ovalling mode shapes for the thin-walled empty silos (diameter $D=$ 
$5.5 \mathrm{~m}$ and wall thickness $t_{\mathrm{s}}=0.07 \mathrm{~m}-0.10 \mathrm{~m}$ varying along the height of the silo) are referred to by a couple $(m, n)$ where $m$ denotes the half wave number in the axial direction and $n$ is the number of circumferential waves (figure 2).

(a)

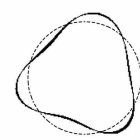

(b)

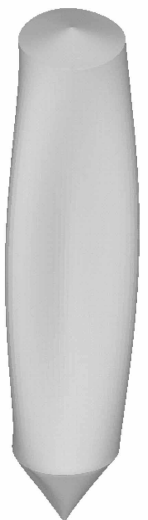

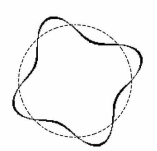

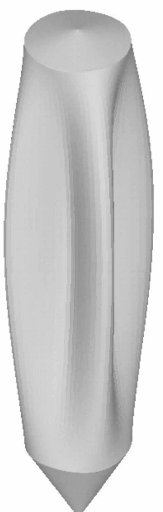

(c)
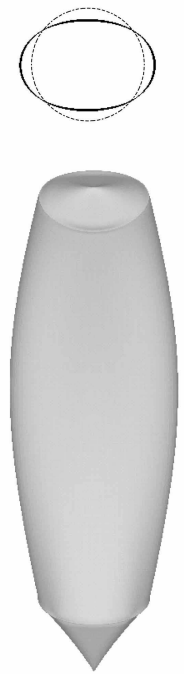

Figure 2: Selected ovalling eigenmodes of a single silo: (a) mode $\boldsymbol{\Phi}_{1}=(1,3)$ at $3.93 \mathrm{~Hz}$, (b) mode $\boldsymbol{\Phi}_{3}=(1,4)$ also at $3.93 \mathrm{~Hz}$ and (c) mode $\boldsymbol{\Phi}_{11}=(1,2)$ at $7.76 \mathrm{~Hz}$ [3].

The governing equations for the structural problem are defined as follows:

$$
\mathbf{M} \ddot{\mathbf{U}}(t)+\mathbf{C} \dot{\mathbf{U}}(t)+\mathbf{K U}(t)=\mathbf{P}(t)
$$

where $\mathbf{M}, \mathbf{C}$ and $\mathbf{K}$ are the mass, damping and stiffness matrix respectively, $\mathbf{U}(t)$ represents the structural displacements and $\mathbf{P}(t)$ are the external loads on the structure. Rayleigh damping is assumed for the damping matrix $\mathbf{C}=\alpha_{\mathrm{R}} \mathbf{M}+\beta_{\mathrm{R}} \mathbf{K}$ with $\alpha_{\mathrm{R}}=0.186 \mathrm{~s}^{-1}$ and $\beta_{\mathrm{R}}=3.03 \times 10^{-4} \mathrm{~s}$, based on a constant damping ratio $\xi=0.75 \%$ for the two lowest eigenmodes. This is a realistic approximation since modal damping ratios $\xi$ for this specific structure, determined during on site measurements by Dooms et al. [6], were found to vary between $0.07 \%$ and $1.32 \%$. These low values of modal damping are typical for a welded aluminium structure.

A FE approach is used to discretize the governing structural equations in the Abaqus software package [3]. To accommodate an easy transfer of the aerodynamic pressures on the silo walls to the mesh of the structural model in the coupled simulations (section 4), the mesh of the FE model was chosen conforming to the mesh on the silo walls in the 3D CFD simulations (figure 4). Since the cone at the bottom of the silo structures is covered by a prismatic building below the silo, this part of the structure is not exposed to the wind flow. A separate mesh was defined for this part of the structure, compatible with that of the superstructure. Shell elements with linear FE interpolation functions are used for all silo elements and the following material properties for aluminium are used: density $\rho=2700 \mathrm{~kg} / \mathrm{m}^{3}$, Young's modulus $E=67.6 \mathrm{GPa}$ and Poisson's ratio $\nu=0.35$. The silo structures are connected to a steel framework, covered by the prismatic building below, at 4 discrete points along the circumference of the cylindrical part of the silo. The mode shapes and natural frequencies of the silo structure are then found by solving the following generalized eigenvalue problem:

$$
\mathbf{K} \Phi=\omega^{2} \mathbf{M} \Phi
$$


where $\omega=2 \pi f_{\text {eig }}$ with $f_{\text {eig }}$ the eigenfrequencies of the structure. The mass normalized eigenmodes $\Phi$ of this eigenvalue problem with the lowest corresponding eigenfrequencies are summarized in table 1. Note that most of the mode shapes come in pairs: e.g. $\boldsymbol{\Phi}_{1}$ and $\boldsymbol{\Phi}_{2}$ are both classified as mode shapes $(1,3)$ but are mutually orthogonal.

\begin{tabular}{ccc}
$\boldsymbol{\Phi}_{j}$ & $(m, n)$ & $f_{\text {eig }}[\mathrm{Hz}]$ \\
\hline $\boldsymbol{\Phi}_{1,2}$ & $(1,3)$ & 3.93 \\
$\boldsymbol{\Phi}_{3,4}$ & $(1,4)$ & 3.93 \\
$\boldsymbol{\Phi}_{5,6}$ & $(1,5)$ & 5.28 \\
$\boldsymbol{\Phi}_{7,8}$ & $(1,5)$ & 5.59 \\
$\boldsymbol{\Phi}_{9,10}$ & $(1,6)$ & 7.38 \\
$\boldsymbol{\Phi}_{11}$ & $(1,2)$ & 7.76
\end{tabular}

Table 1: Structural natural frequencies $f_{\text {eig }}$ of ovalling mode shapes of the silo structure.

The visually detected pattern of vibrations at the lee side of the silo group during the 2002 storm are believed to have been ovalling mode shapes $(1,3)$ and $(1,4)$, with the lowest natural frequencies of the silo structure. Measurements during normal wind loading have also shown that eigenmodes with 3 or 4 circumferential wavelengths have the highest contribution to the response of the silos [6].

\section{WIND FLOW SIMULATIONS}

The highly turbulent wind flow around a single silo is investigated by performing 3D CFD simulations. The numerical procedure, computational domain and issues concerning the boundary conditions are discussed first. For validation purposes, a comparative study of the aerodynamic pressures determined in the simulations and measured in a wind tunnel experiment is performed. Finally, the flow pattern around the single silo is discussed and qualitatively compared with available literature.

\subsection{Numerical procedure}

The governing incompressible Navier-Stokes equations are discretized by means of the finite volume method in the CFD simulations. Since the focus in this analysis is on the structural response, it is unnecessary to resolve all details of turbulent fluctuations in the flow. Instead of resolving all turbulent scales in a direct numerical simulation (DNS), other numerical techniques were developped for the treatment of turbulence in the simulations, e.g. Reynolds averaged Navier-Stokes (RANS) simulations or large eddy simulations (LES).

For the highly turbulent, external aerodynamic flow of the present case $\left(\operatorname{Re}=1.24 \times 10^{7}\right)$, DNS or LES simulations are computationally too demanding, especially since accurate nearwall flows are required to get a good prediction of the aerodynamic pressures on the silo walls. It is therefore advisable to use the near-wall modeling of the RANS techniques. In this light, delayed detached eddy simulations (DDES) are performed in Ansys Fluent [1].

DES models are refered to as hybrid LES/RANS models because the URANS modeling of the boundary layer flow in the near-wall region is combined with the LES approach in the separated regions, where large unsteady turbulence scales are dominant. In the delayed DES approach, a shielding function is used to ensure that RANS is applied in the entire boundary layer since a sole geometrical separation of RANS and LES regions based on mesh size has been shown to be insufficient. For the shielding function, the blending functions of the SST 


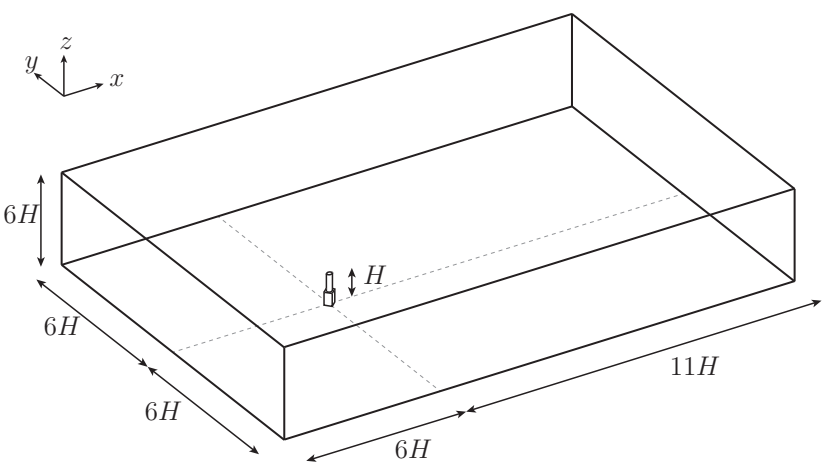

Figure 3: Dimensions of the 3D computational domain and global coordinate system, with origin at the bottom of the domain at the center of the structure.

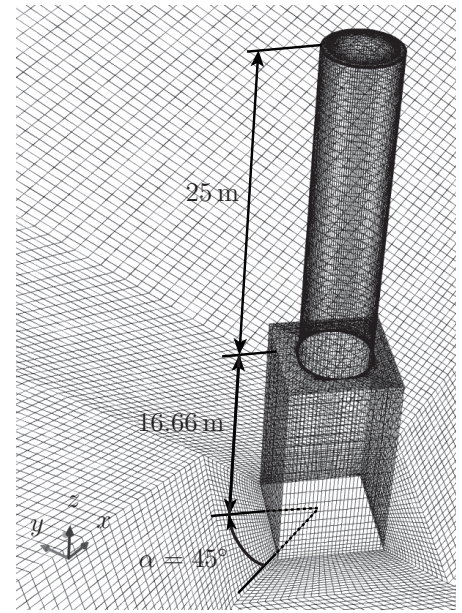

Figure 4: Detail of the mesh for 3D DDES of a single silo structure with the wind at an angle of incidence $\alpha=$ $45^{\circ}$.

turbulence model are used [10]. Coupled pressure-based simulations with a second-order interpolation of the pressure, a second-order upwind interpolation of the turbulent kinetic energy $k$ and the specific dissipation rate $\omega$ and a second-order implicit, unconditionally stable, time stepping method are performed. For the discretization of the momentum equations a bounded central differencing scheme is used in DDES.

\subsection{Computational domain and boundary conditions}

The boundaries of the computational domain should be sufficiently far from the zone of interest in the centre of the domain (i.e. where the silo structures are located). Several guidelines are available in the literature with rules of thumb for the size of the computational domain and the boundary conditions.

Based on wind tunnel experiments, guidelines have been set up by the Architectural Institute of Japan (AIJ) for the size of the computational domain in 3D wind simulations [18]. For a single building model, the lateral and the top boundaries should be set $5 \mathrm{H}$ or more away from the building and the outflow boundary should be located at least $10 \mathrm{H}$ behind the building where $H$ is the height of the building. Furthermore, the blocking ratio (i.e. building cross section/domain section) should be below 3\%. As shown in figure 3, slightly larger values of $6 \mathrm{H}$ and $11 \mathrm{H}$ are used, resulting in a blocking ratio of $1.7 \%$. The outflow boundary is modeled as a pressure outlet while symmetry is imposed on lateral and top boundaries. The walls of the structure are considered smooth and no-slip boundary conditions are applied.

Since the specific atmospheric conditions near the silo group were not monitored during the storm, approximative wind conditions have to be set up, based on the location of the group and mean wind velocities for storm conditions in design codes. Based on the Eurocode 1 design guidelines for wind loading [5], a mean wind velocity $v_{\infty}=31.8 \mathrm{~m} / \mathrm{s}$ is determined at half the height of the silos (approx. $z=30 \mathrm{~m}$ ), resulting in a post-critical wind flow at Reynolds number $\operatorname{Re}=v_{\infty} D / \nu=1.24 \times 10^{7}$. Furthermore, the logarithmic velocity profile and realistic turbulence profiles of the atmospheric boundary layer (ABL) have to be imposed at the inlet of the 3D computational domain. As recommended by AIJ [18], a power law is used in the 
simulations:

$$
\begin{aligned}
v_{x}(z) & =v_{\mathrm{R}}\left(z / z_{\mathrm{R}}\right)^{\alpha} \\
\mathrm{Tu}(z) & =0.1\left(z / z_{\mathrm{G}}\right)^{-\alpha-0.05}
\end{aligned}
$$

where $v_{\mathrm{R}}=v_{\infty}=31.8 \mathrm{~m} / \mathrm{s}$ is the reference velocity at a reference height $z_{\mathrm{R}}=30 \mathrm{~m}$. The exponent $\alpha=0.14$ and gradient height $z_{\mathrm{G}}=300 \mathrm{~m}$ are determined for terrain category 2 (open country) according to the AIJ guidelines [18]. Based on these inlet boundary conditions and standard relations in fluid mechanics, inlet profiles for turbulent kinetic energy $k$ and specific dissipation rate $\omega$ can be derived and imposed at the inlet. To simulate time dependent fluctuations superimposed on these mean profiles, a spectral synthesizer method is used [16].

\subsection{Experimental validation}

Apart from the verification of the numerical procedure (grid size refinement and time step refinement), which is not discussed in detail in this paper, it is very important that the simulation results are also validated with some experimental data or full-scale measurements. Because of the high Reynolds number and the particular geometry of this single silo case (figure 4), no such data are available in the literature. An experimental set-up of the present geometry was therefore prepared in a wind tunnel.

In view of the present purposes, the experimental validation is primarily aimed at a comparison of the aerodynamic pressure distribution on the silo surface. Pressures on the square prismatic building below the silo stucture and other parameters of interest (e.g. velocities in the wake flow etc.) are not considered in this paper.

\subsubsection{Experimental apparatus and techniques}

The experiments were carried out in the fluid mechanics laboratory at the Department of Mechanical Engineering at the Vrije Universiteit Brussel (VUB), using the wind tunnel 1 facility. This open circuit wind tunnel is mainly designed for civil engineering experimental setups and the relatively high testing chamber ( $2 \mathrm{~m}$ wide, $1 \mathrm{~m}$ high and $12 \mathrm{~m}$ long) allows conducting measurements with different geometry shapes. The dimensions of the scaled model are calculated by optimizing the cross section area of the model inside the wind tunnel. This method leads to a compromise between blockage effect reduction and Reynolds number increase. The resulting scale factor is 1:50 and the model geometry is rotated $45^{\circ}$ with respect to the flow direction, similarly as in the CFD simulations of the single silo. No roughness elements were placed in the wind tunnel section to simulate a natural boundary layer.

The scaled model consists of two different compartments: the building part where no probes are installed (wooden box), and a cylindrical PVC tube including the pressure sensor system (figure 5a). The free end of the PVC tube is finished with a conical PVC top to match the silo geometry. The measurement points in the cylindrical part are distributed uniformly along the height of the tube (figure 6). At each point, a pressure tap (metallic needle) is installed through the tube shell in order to capture the (unsteady) static pressure on its surface (figure 5b). The installation of these pressure taps is particularly critical. The silo surface should have the smallest possible discontinuities and the needles need to be installed perpendicular to the surface to minimize the effect of dynamic pressure contribution.

A Scanivalve pressure measurement device [2] is installed in the wooden building compartment of the model and is connected to all pressure taps via flexible tubes. For this set of experiments a 64-channel valve-less piezoelectric device is used that communicates the unsteady 
(a)

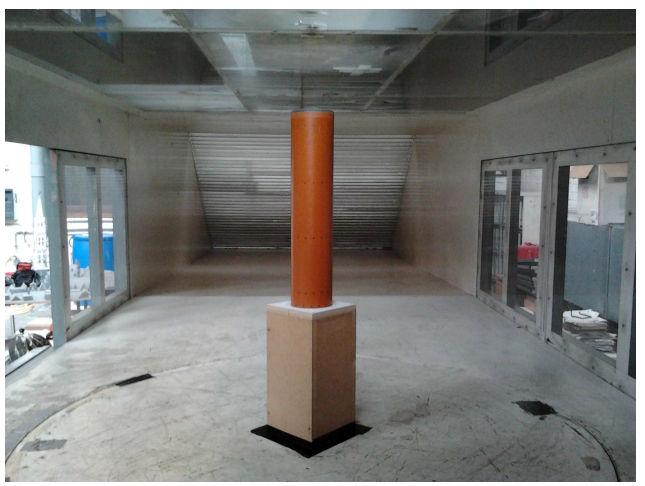

(b)

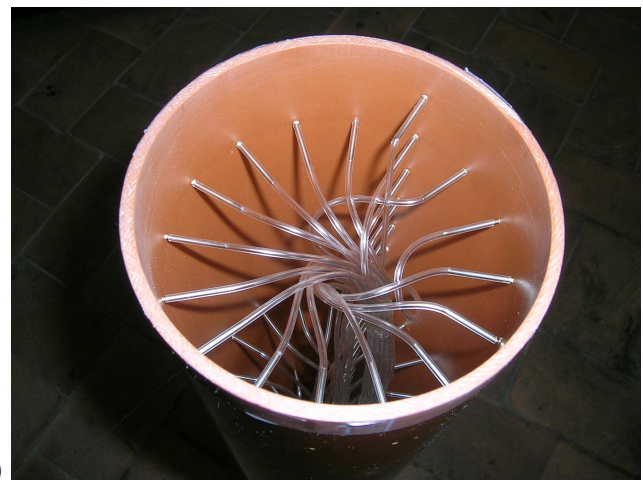

Figure 5: Set up of the wind tunnel experiments: (a) downstream view of the scale model of the silo structure in the wind tunnel and (b) pressure tabs installed in the interior of the PVC tube.

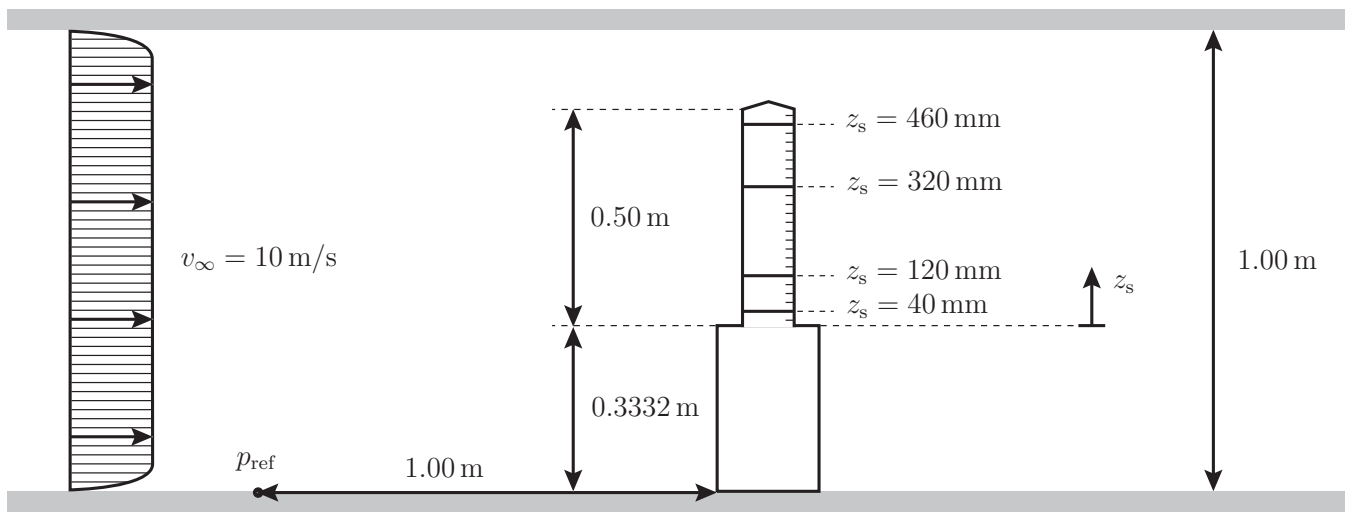

Figure 6: Schematic representation of wind tunnel test section.

pressure data to a computer, using a channel-multiplexing method. The ability to measure multiple channels at high frequencies up to $2 \mathrm{kHz}$ is one of the main advantages of this device. A set of 24 pressure taps is installed, aligned and equally distributed along the vertical axis of the cylinder (figure 5). The cylindrical tube is installed in such a way that the complete pressure distribution on the surface can be measured by rotating the entire cylinder around its axis of symmetry in 16 rotational steps $\left(22.5^{\circ}\right)$.

The experiments are carried out in the ambient conditions of the lab. The Scanivalve device measures the differential pressure with respect to a reference point. In this case, the static pressure is measured in a reference point situated $1 \mathrm{~m}$ upstream of the model at the bottom of the wind tunnel test section (figure 6). All possible fluctuations of air humidity and temperature are neglected during the measurements.

\subsubsection{Experimental and numerical set-up}

For a good comparison of experimental and numerical results, the wind tunnel flow should have similar characteristics as the numerically simulated natural wind around the structure or vice versa. Furthermore, reduced geometric scales are typically used in wind tunnel experiments for obvious reasons of economy and convenience. Such scaling operation introduces the question of physical simulitude for which a set of dimensionless numbers and/or similarity criteria are applicable to both flow and structure. Numerous works have been published describing these similarity requirements, e.g. [15]. 
In the present study, however, dimensional analysis and difficulties to achieve similarity between wind tunnel and real atmospheric flows have been avoided by adapting the wind tunnel experiments and the simulations to each other. On the experimental side, a model was constructed corresponding to the geometry of the single silo. The size of this model had to be small enough in order to fit the wind tunnel section and large enough so that it could be instrumented with the pressure tabs. The numerical simulations in their turn were scaled down to the exact size of the wind tunnel experiment (i.e. scaling of 1:50) but leaving the dimensions of the computational domain (cfr. figure 3) and the mesh refinement unchanged with respect to the structure size. These operations have several important consequences for the validation:

- The wind velocities that can be reached in the wind tunnel are not nearly large enough to simulate a wind flow at a Reynolds number similar to that in the full scale numerical simulations $\left(\operatorname{Re}=1.24 \times 10^{7}\right)$, described in section 3.2. Instead, a velocity of $10 \mathrm{~m} / \mathrm{s}$ is applied at the inlet of the wind tunnel and also in the numerical simulations. The resulting Reynolds number $\left(\operatorname{Re}=6.25 \times 10^{4}\right)$ categorizes the wind flow in the subcritical flow regime (transition in the shear layer) as opposed to the post-critical regime (fully turbulent shear layer and wake) in the real-scale simulations at higher wind velocities and Reynolds number [15, 19]. This should be taken into account for the interpretation of the simulation results because different physical phenomena are to be expected in the transitional and post-critical regime, especially in the behaviour of the attached shear layer.

- Due to the vicinity of the top wall of the wind tunnel (figure 6), some blockage effects are to be expected in the pressure measurements in the upper part of the silo structure. In the simulations, the top of the computational domain is much more distant from the free end of the silo structure and pressures might differ.

These discrepancies make the present comparison not entirely representative as validation for the single silo in cross flow. However, taking into account the above mentioned considerations in the comparison of the results, this validation is nevertheless a valuable asset to increase the confidence in the numerical simulations.

\subsubsection{Comparison of experimental and numerical results}

As mentioned, only the pressure distribution on the silo structure will be reviewed in the validation study. Both mean pressures and root-mean square (RMS) values of the surface pressures are shown in figure 7 at four locations along the height of the cylinder surface.

At the windward side of the cylinder, the mean pressures agree very well in the attached flow from the stagnation point until separation is reached. In the simulations, separation occurs later than in the experiments, leading to lower negative base pressures at the lee side of the cylinder. This difference between simulations and experiments in the prediction of the separation point and consequently the base pressures at the lee side, can be explained by considering the applied turbulence model in the simulations.

In the DDES simulations, the SST turbulence model [9] is used for the RANS solution in the near-wall regions. This turbulence model is typically very efficient for fully turbulent boundary layers at high Reynolds numbers but switches to turbulent modelling of the boundary layer flow too quickly at lower Reynolds numbers. The separation point is consequently not captured accurately in the simulation for subcritical flow in the experiments and a narrower 
(a)
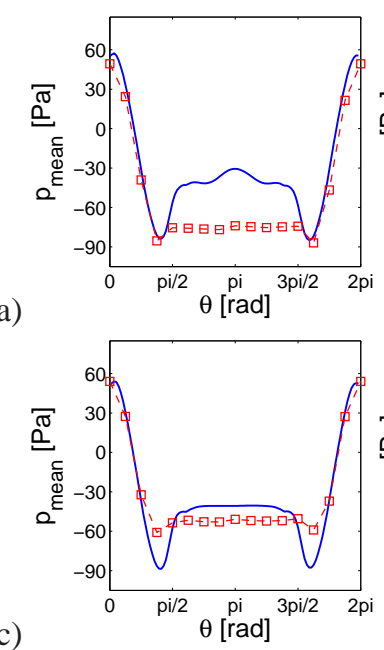
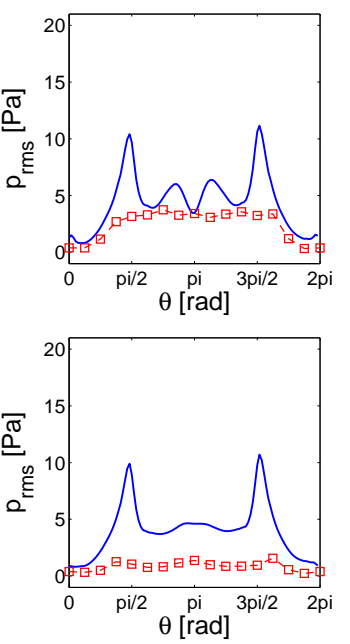

(b)
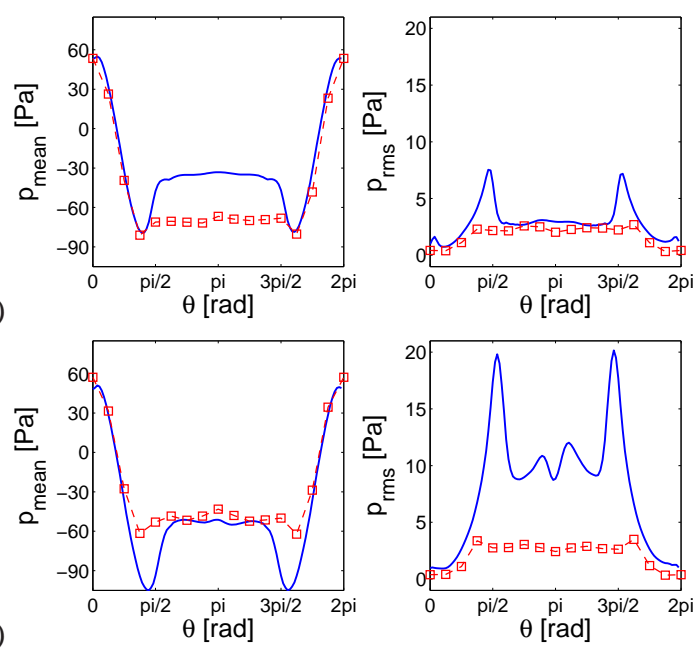

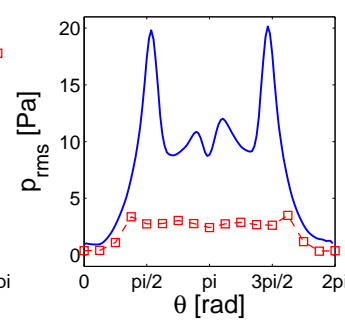

Figure 7: Experimental ( $\square$, dashed line) and numerical (full line) mean pressure and RMS pressure distribution along the circumference of the cylindrical silo structure for four different locations along the height of the silo: (a) $z_{\mathrm{s}}=40 \mathrm{~mm}$, (b) $z_{\mathrm{s}}=120 \mathrm{~mm}$, (c) $z_{\mathrm{s}}=320 \mathrm{~mm}$, and (d) $z_{\mathrm{s}}=460 \mathrm{~mm}$.

wake is formed at the lee side of the cylinder, typical for post-critical flows. Unfortunately, no DES simulations can be performed with a more suitable transitional turbulence model in the RANS part and on the other hand, no wind tunnel simulations can be performed up to Reynolds numbers high enough to capture a fully turbulent boundary layer on the cylinder surface. Nevertheless, for the present full-scale application with post-critical wind flow, the applied numerical turbulence model is believed to yield accurate results.

Furthermore, although experiments and simulations seem to generate comparable pressure profiles near the bottom part of the cylinder surface (figure 7 $\mathrm{a}, \mathrm{b}$ ), the agreement seems to decrease moving towards the free end of the cylinder (figure 7k, d). This discrepancy is attributed to blockage effects due to the vicinity of the top wall of the wind tunnel near the free end of the structure (figure 6).

The conclusions for the mean pressures can be extended to the fluctuating pressures (RMS) as well. The agreement is good at the windward side of the structure while the separation point is delayed in the simulations, resulting in higher fluctuating pressure peaks. However, the agreement of the fluctuating pressures at the leeward side of the cylinder seems to be better than for the mean pressures. The leeward pressure fluctuations are of the same order of magnitude in experiments and simulations, although the agreement deteriorates when moving upwards. This is in agreement with the observations for the mean pressures.

\subsection{Flow pattern around the single silo}

To gain physical insight in the flow pattern around the present single silo structure in the natural wind conditions $\left(\operatorname{Re}=1.24 \times 10^{7}\right)$, it is instructive to compare the simulated flow pattern with that around similar surface-mounted bluff bodies in cross flow. Furthermore, apart from the quantitative validation discussed in section 3.3, such qualititave comparison increases the confidence in the simulation results.

The present geometry can be considered as the combination of two different types of bluff bodies: a cylinder is placed on top of a square prism. A reasonable amount of literature can be found where the flow around cantilevered cylinders [4, 12, 13] and prismatic obstacles [7, 14, 
17] is investigated experimentally. However, as for the wind tunnel experiment in this paper, Reynolds numbers in the experimental set-ups in the literature are typically in the order of magnitude of $10^{4}-10^{5}$. Great care should therefore been taken when comparing the present flow pattern with experimental flow visualization studies in the literature.

Due to the highly 3D nature of the flows, different flow patterns coexist over different spanwise positions of the silo structure. For this purpose the streamlines of the flow are shown along a vertical plane in figure 8 and in 5 horizontal planes across the height of the silo structure in figure 9 .

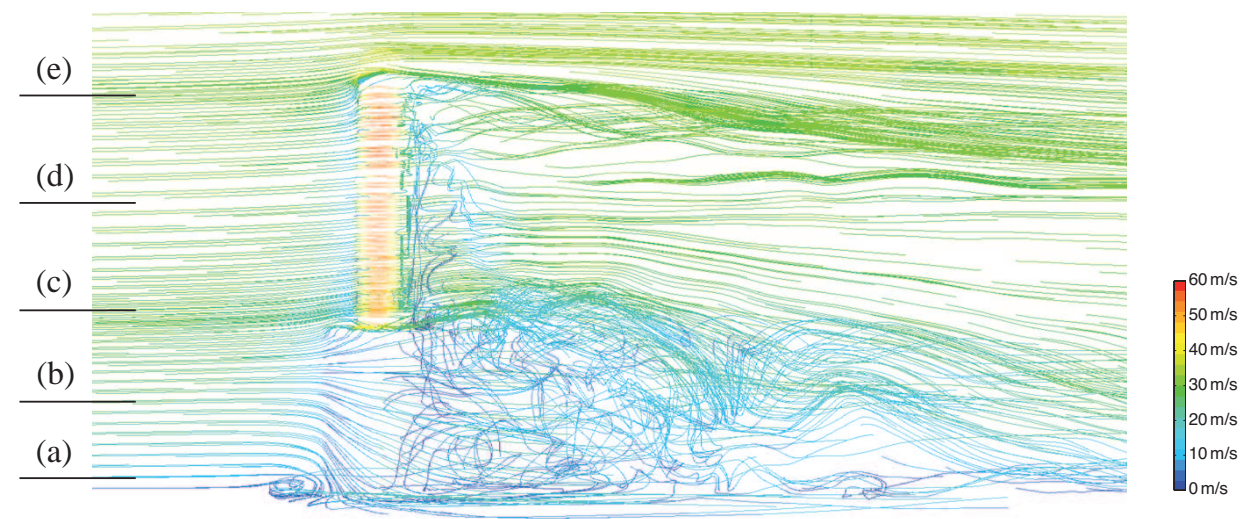

Figure 8: Velocity streamlines of the wind flow around a single silo structure at $t=70 \mathrm{~s}$, colored according to the velocity magnitude, in a vertical plane $y=0 \mathrm{~m}$. Reference is made to the 5 horizontal planes shown in figure 9 .

(a)
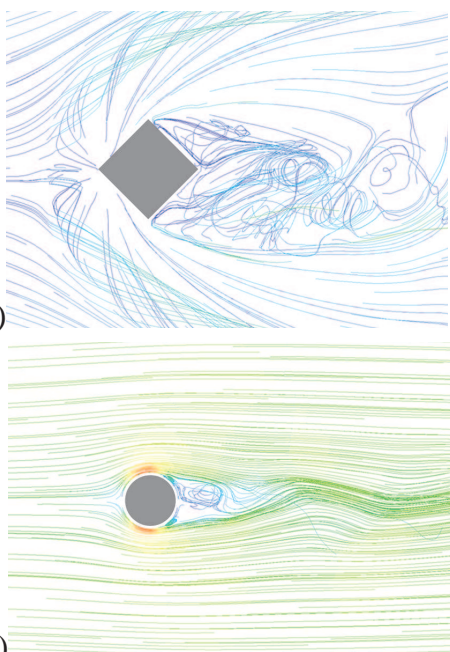

(b)
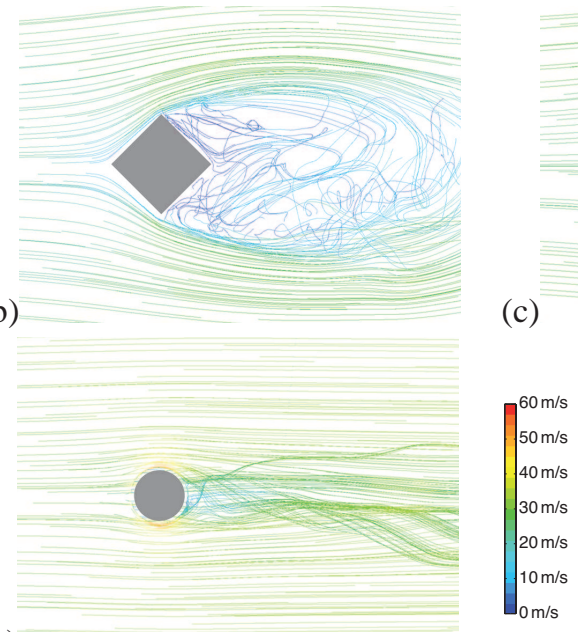

(e)

(c)

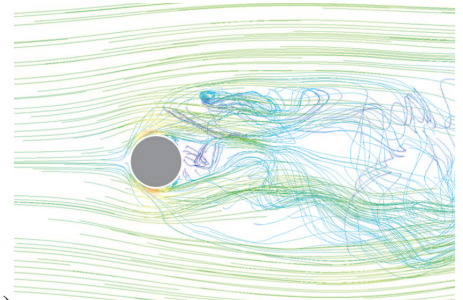

Figure 9: Velocity streamlines of the wind flow around a single silo structure at $t=70 \mathrm{~s}$, colored according to the velocity magnitude, in horizontal planes at (a) $z=0.5 \mathrm{~m}$, (b) $z=8 \mathrm{~m}$, (c) $z=17.16 \mathrm{~m}$, (d) $z=29.16 \mathrm{~m}$, and (e) $z=41.16 \mathrm{~m}$.

At the base of the silo structure, the wind flow is forced around the square prismatic building which is rotated at an angle of $45^{\circ}$ with respect to the incident wind flow. As observed in the literature [4], upstream of an isolated surface-mounted structure, the natural turbulent boundary layer on the surface undergoes a three-dimensional separation and the lower regions of the 
separated boundary layer roll up to form a vortex system upstream of the building (figure 8). The ends of this vortex system are swept downstream and the typical horseshoe (or necklace) vortex is formed. This behaviour is observed for both prismatic [14, 17] and cylindrical [4] cantilevered structures. In figure $9 \mathrm{a}$, the formation of a horseshoe vortex upstream of the obstacle is also observed in the present simulations at much higher Reynolds number.

At mid-span of the square prismatic building below the silo structure, the flow near the lateral upstream faces of the structure are mainly stable and no flow separation is observed (figure 9b). The attached flow separates at the transverse corners of the square prism. At the lee side, a turbulent wake is produced between the separated shear layers.

At the connection of the square prismatic building to the cylindrical silo structure, the flow is slightly accelerated and deflected upwards along the upper corners of the lateral upstream sides (figure 8). The flow separates and is simultaneously deflected sideways, parried by the cylindrical silo structure (figure 9r).

The flow pattern around the upper silo structure resembles that around a cantilevered cylinder in cross flow. In this part of the structure, 3D flow effects are mainly attributed to the flow separation and resulting downwash flow near the free end of the cylinder. Nonetheless, a suppressed 2D region can exist along the cylinder height, even with vortex shedding resembling the von Kármán vortex street at lower Reynolds numbers [8]. In figure 9d, the streamlines at mid-span of the cylindrical structure are shown. The flow is separated at the lee side of the cylindrical silo surface, generating a highly turbulent and narrow wake region, typical for high Reynolds number cross flows around cylinders [15, 19].

Near the top of the cylinder (figures 8 and 9 e), the approaching flow moves upward, accelerates and then separates from the cylinder circumference at the free end to form a trailing vortex. No large recirculation zones are observed at the top of the cylindrical silo and the separated trailing vortex is mainly dragged downstream and only slightly deflected downwards. The limited downwash at the lee side of the cylinder also results in minor interaction with the flow at midspan of the silo.

For lower Reynolds number flows (e.g. $2 \times 10^{4}$ in [12]), two counter-rotating vortices are formed above the free end of the cylinder which subsequently descend along the central section of the wake. The vortices expand laterally, move slightly downwards and interact with the vortices shed from the two sides of the cylinder in the upper half of the near-wake region of the cylinder [12]. Finally, Park et al. [13] found that a modification of the free end geometry of the cylinder (e.g. a bevelled or radiussed free end) can reduce the width of the wake formed behind the finite cylinder. This narrow wake region is also observed in the present simulations (figures 9d and 9e) but is of course also related to the higher Reynolds number flow in the present application.

\section{WIND-STRUCTURE INTERACTION SIMULATIONS FOR A SINGLE SILO}

With a numerical model available for both the structure and the wind flow, it is now possible to consider the coupled numerical problem as a whole. In the wind-structure interaction simulations, the solvers remain separated and the interaction between both domains is only active at the interface between structure and wind flow. The structural solver can be denoted as follows:

$$
\mathcal{S}[\mathbf{P}(t)]=\mathbf{U}(t)
$$

where $\mathbf{U}(t)$ are the displacements of the structure and $\mathbf{P}(t)$ are the aerodynamic pressures acting on the structure. This expression corresponds with the numerical solution of the structural 
governing equation (equation 1). Similary, the numerical solver of the Navier-Stokes equations of the wind flow can be denoted as:

$$
\mathcal{F}[\mathbf{U}(t)]=\mathbf{P}(t)
$$

As mentioned in the introduction, two different coupling approaches are considered as schematically represented in figure 10. Only partitioned techniques are being considered here, implying that both the structural and flow solver are maintained as separated solvers (e.g. two black-box solvers) with only interaction at the wind-structure interface.

In the one-way coupling approach (figure $10 \mathrm{a}$ ), the structure is considered as a rigid body in the wind flow simulations: $\mathbf{U}\left(t_{i}\right)=\mathbf{0}$. In every time step, the aerodynamic surface pressures on the rigid body structure can be determined in the flow solver: $\mathcal{F}[\mathbf{0}]=\mathbf{P}\left(t_{i}\right)$. Subsequently, the resulting time history of surface pressures $\mathbf{P}(t)$ is applied as an external transient load on the structure and the resulting structural displacements can be computed: $\mathcal{S}[\mathbf{P}(t)]=\mathbf{U}(t)$. To avoid interpolation of surface pressures on the interface, the computational grid of the silo surface was made identical in flow and structural solver. This way, the aerodynamic pressures and structural displacements on the wind-structure interface can be transferred between solvers with a node-to-node algorithm, without further need of approximations through interpolation.

In the two-way coupling approach, on the contrary, the structural and flow solver are coupled in every time step (figure $10 \mathrm{~b}$ ). To ensure equilibrium at the fluid-structure interface in every time step, several Gauss-Seidel coupling iterations between solvers are performed. In the first coupling iteration (superscript ${ }^{1}$ ) the aerodynamic surface pressures are calculated in the flow solver $\mathcal{F}\left[\mathbf{U}^{*}\left(t_{i}\right)\right]=\mathbf{P}^{1}\left(t_{i}\right)$ from an extrapolation of the structural displacements from previous time steps, denoted $\mathbf{U}^{*}\left(t_{i}\right)$. Subsequently, the structural response to these aerodynamic pressures is calculated $\mathcal{S}\left[\mathbf{P}^{1}\left(t_{i}\right)\right]=\mathbf{U}^{1}\left(t_{i}\right)$. In the second coupling iteration, feedback is given from the structural to the flow solver where the fluid mesh deforms, the wind flow is slightly modified and new surface pressures can be calculated: $\mathcal{F}\left[\mathbf{U}^{1}\left(t_{i}\right)\right]=\mathbf{P}^{2}\left(t_{i}\right)$. The updated surface pressures in their turn give rise to new structural displacements: $\mathcal{S}\left[\mathbf{P}^{2}\left(t_{i}\right)\right]=\mathbf{U}^{2}\left(t_{i}\right)$. This iterative procedure is repeated until convergence of aerodynamic surface pressures and structural displacements. In the present simulations, five Gauss-Seidel iterations per time step are needed to reach convergence on the wind-structure interface.

It is self-evident that the computational effort to perform a two-way coupled simulation is larger. Although it is difficult to compare the exact amount of computation time required to simulate a single time step in the one-way coupling (where the flow solver and the structural solver have to be set-up separately and the one precedes the other) and the two-way coupling, it takes approximately 5 times longer to execute a two-way coupling simulation. It is therefore interesting to assess the necessity of performing these computationally much more imposing simulations.

\subsection{One-way coupling simulations}

In the one-way coupling simulations, the structural response $\mathbf{U}(t)$ is calculated by applying the previously determined time history of aerodynamic pressures $\mathbf{P}(t)$ on the FE model of the silo. A direct time integration scheme, the unconditionally stable and second order accurate Hilbert-Hughes-Taylor method as implemented in the Abaqus FE solver [3], is used to solve the governing system of dynamic structural equations (equation 1).

To avoid a long period of transitional effects in the structural response, a static calculation 


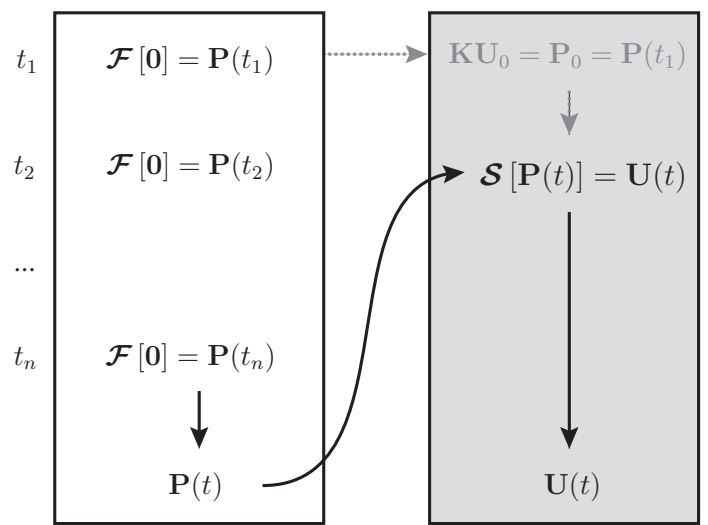

(a)

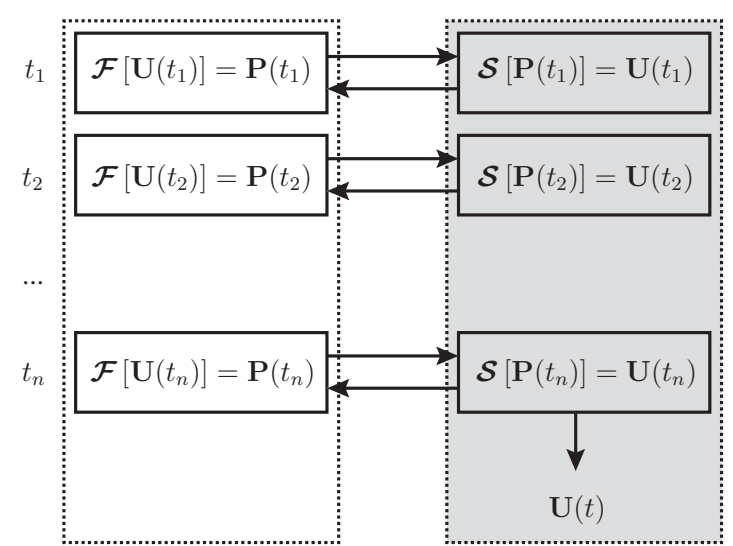

(b)

Figure 10: Schematic representation of the interaction between flow solver (white) and structural solver (grey) in the (a) one-way and (b) two-way partitioned coupling approaches.

precedes the dynamic calculation (cfr. figure 10a):

$$
\mathbf{K U}_{0}=\mathbf{P}_{0}
$$

The applied pressures in this stationary step are taken equal to those of the first dynamic time step: $\mathbf{P}_{0}=\mathbf{P}\left(t_{1}\right)$. The structural response $\mathbf{U}_{0}$ can subsequently be used as an initial condition for the dynamic calculations. The effect of this preliminary static calculation has been verified for the present simulations and found to be very effective.

The calculated structural response $\mathbf{U}(t)$ to the aerodynamic surface pressures, shows peak displacements of about $0.03 \mathrm{~m}$. This value is realistic but relatively high considering the estimated $0.05-0.10 \mathrm{~m}$ of the observed vibrations during the 2002 storm in the Antwerp silo group, based on video footage.

In order to distinguish the contribution of the different mode shapes to the response, modal decomposition techniques are used to determine the kinetic and deformation energy in the structural response. Both the deformation energy $E_{\mathrm{d}}(t)$ and the kinetic energy $E_{\mathrm{k}}(t)$ can be easily calculated from the known structural displacements $\mathbf{U}(t)$ and velocities $\mathbf{U}(t)$ :

$$
E_{\mathrm{d}}(t)=\frac{1}{2} \mathbf{U}^{\mathrm{T}}(t) \mathbf{K} \mathbf{U}(t) \quad E_{\mathrm{k}}(t)=\frac{1}{2} \dot{\mathbf{U}}^{\mathrm{T}}(t) \mathbf{M} \dot{\mathbf{U}}(t)
$$

By applying modal decomposition of the structural deformations $\mathbf{U}(t)=\boldsymbol{\Phi} \boldsymbol{\alpha}(t)$ and velocities $\dot{\mathbf{U}}(t)=\boldsymbol{\Phi} \dot{\boldsymbol{\alpha}}(t)$ where $\boldsymbol{\alpha}(t)$ represent the modal coordinates, the energy content of each structural mode shape in the response can be quantified:

$$
\begin{aligned}
E_{\mathrm{d}}(t) & =\frac{1}{2} \mathbf{U}^{\mathrm{T}}(t) \mathbf{K} \mathbf{U}(t) & E_{\mathrm{k}}(t) & =\frac{1}{2} \dot{\mathbf{U}}^{\mathrm{T}}(t) \mathbf{M} \dot{\mathbf{U}}(t) \\
& =\frac{1}{2} \boldsymbol{\alpha}^{\mathrm{T}}(t) \boldsymbol{\Phi}^{\mathrm{T}} \mathbf{K} \boldsymbol{\Phi} \boldsymbol{\alpha}(t) & & \frac{1}{2} \dot{\boldsymbol{\alpha}}^{\mathrm{T}}(t) \boldsymbol{\Phi}^{\mathrm{T}} \mathbf{M} \boldsymbol{\Phi} \dot{\boldsymbol{\alpha}}(t) \\
& =\frac{1}{2} \sum_{j=1}^{N} \omega_{j}^{2} \alpha_{j}^{2}(t) & & \frac{1}{2} \sum_{j=1}^{N} \dot{\alpha}_{j}^{2}(t) \\
& =\sum_{j=1}^{N} E_{\mathrm{d}, j}(t) & & =\sum_{j=1}^{N} E_{\mathrm{k}, j}(t)
\end{aligned}
$$


Based on these scalar energy expressions where $N$ represents the total number of mode shapes, the energy contribution $E_{\mathrm{d}, j}(t)$ and $E_{\mathrm{k}, j}(t)$ of every seperate mode shape $j$ to the structural response can be determined using only the modal coordinates $\boldsymbol{\alpha}(t)$ and $\dot{\boldsymbol{\alpha}}(t)$.

However, in the present form of equations 9 , the entire basis of $N$ mode shapes $\Phi$ would have to be determined to extract the modal coordinates $\boldsymbol{\alpha}(t)$ from the known structural displacements $\mathbf{U}(t)$. It is however computationally very inefficient to solve the entire eigenvalue problem (equation 2) for the determination of $\Phi$. Furthermore, only the lowest eigenmodes are relevant in the dynamic response of the structure for a typical low frequency wind excitation. It is therefore desirable to use only a subset $\boldsymbol{\Phi}_{\mathrm{s}}$ with corresponding modal coordinates $\boldsymbol{\alpha}_{\mathrm{s}}(t)$ to determine the deformation and kinetic energy, where $\boldsymbol{\Phi}=\left[\boldsymbol{\Phi}_{\mathrm{s}} \mid \boldsymbol{\Phi}^{\prime}\right]$ and $\boldsymbol{\alpha}(t)=\left\{\begin{array}{l}\boldsymbol{\alpha}_{\mathrm{s}}(t) \\ \boldsymbol{\alpha}^{\prime}(t)\end{array}\right\}$.

Of course, while $\mathbf{U}(t)=\boldsymbol{\Phi} \boldsymbol{\alpha}(t)$ is always true, an alternative projection $\hat{\boldsymbol{\alpha}}_{\mathrm{s}}(t)$ has to be proposed to approximate $\boldsymbol{\alpha}_{\mathrm{s}}(t)$ so that $\mathbf{U}(t)=\boldsymbol{\Phi}_{\mathrm{S}} \hat{\boldsymbol{\alpha}}_{\mathrm{s}}(t)$. It can easily be shown by relying on the orthogonality property of the structural mass matrix that the modal coordinates $\boldsymbol{\alpha}_{\mathrm{s}}(t)$ can be exactly calculated by using the following projection method:

$$
\boldsymbol{\alpha}_{\mathrm{s}}(t) \equiv \hat{\boldsymbol{\alpha}}_{\mathrm{s}}(t)=\boldsymbol{\Phi}_{\mathrm{s}}^{\mathrm{T}} \mathbf{M U}(t)
$$

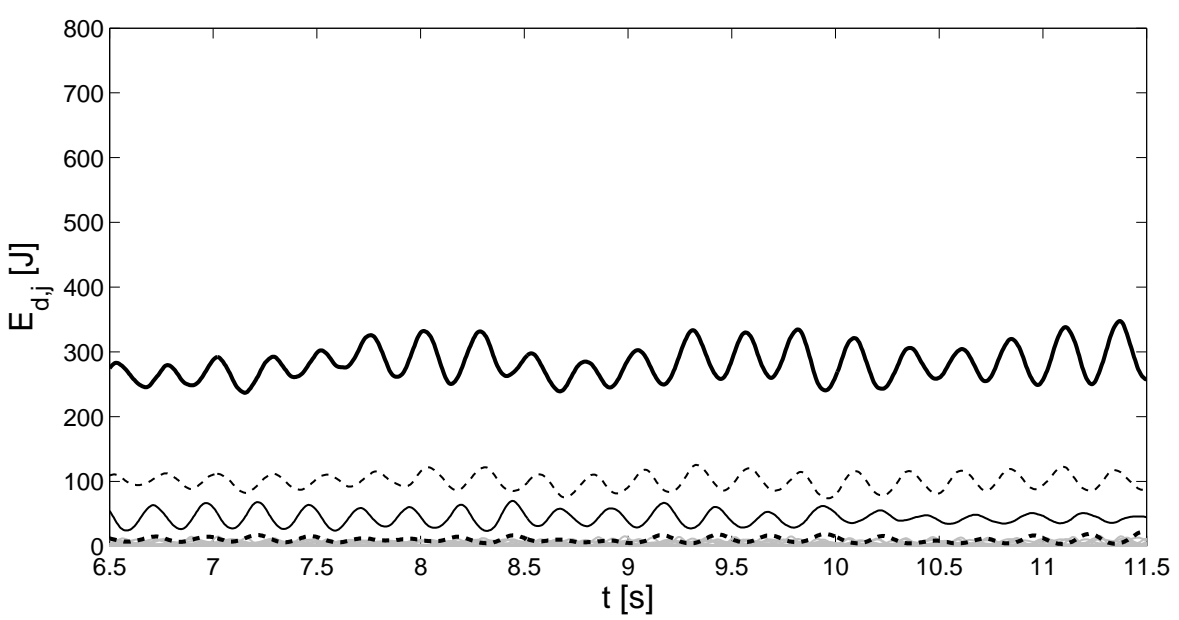

Figure 11: Modal deformation energy $E_{\mathrm{d}, j}$ for the first 20 mode shapes, based on the structural response in the one-way coupling simulation: $\boldsymbol{\Phi}_{1}=(1,3)$ (dashed bold black line), $\boldsymbol{\Phi}_{2}=(1,3)$ (solid bold black line), $\boldsymbol{\Phi}_{3}=(1,4)$ (dashed thin black line), $\boldsymbol{\Phi}_{4}=(1,4)$ (solid thin black line), and the remaining mode shapes $\boldsymbol{\Phi}_{j}$ (solid light grey lines, with small energy content).

Figure 11 shows the modal deformation energy $E_{\mathrm{d}, j}(t)$ for the first 20 mode shapes. Only mode shapes $\boldsymbol{\Phi}_{1}=(1,3), \boldsymbol{\Phi}_{2}=(1,3), \boldsymbol{\Phi}_{3}=(1,4)$ and $\boldsymbol{\Phi}_{4}=(1,4)$ have a significant contribution to the structural response of the silo. The contribution of other mode shapes is distinctly smaller. The deformation energy of the first three mode shapes mentioned has an important static (time averaged) component indicating that these mode shapes contribute significantly to the static deformation of the silo structure. The deformation energy of all these excited mode shapes is oscillating at about $4 \mathrm{~Hz}$, coinciding with their natural frequencies and hence indicating a dynamic response in mode shapes $(1,3)$ and $(1,4)$. After transformation to the frequency domain of the modal deformation energy $E_{\mathrm{d}, j}(f)$, the excitation at the natural frequencies is 
clearly confirmed (figure 12). However, smaller and irregular low frequency fluctuations can be observed as well, especially for the mode shapes with the largest static excitation. These low frequency oscillations are also visible in figure 11 and are inherent to the simulated low frequency content wind turbulence. These oscillations can be seen as a 'quasi-static' sway about the mean static response, depending on and following the turbulent fluctuations of the incoming wind flow.

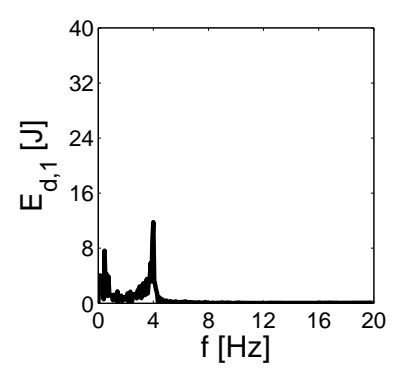

(a)

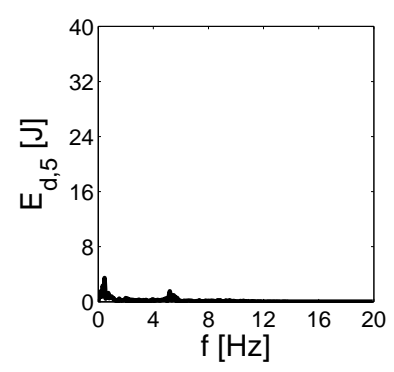

(e)

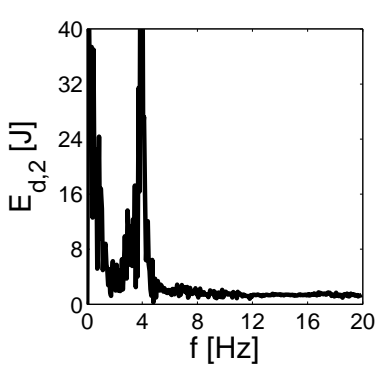

(b)

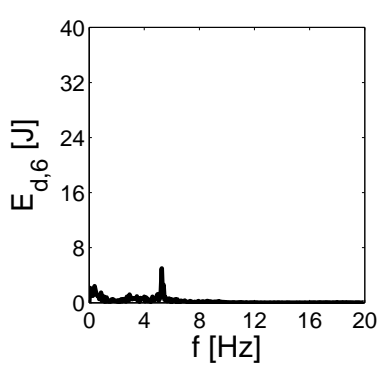

(f)

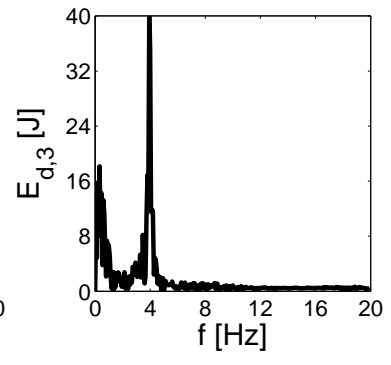

(c)

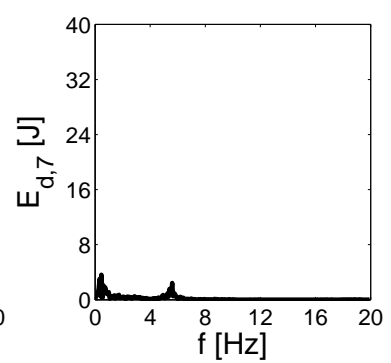

(g)

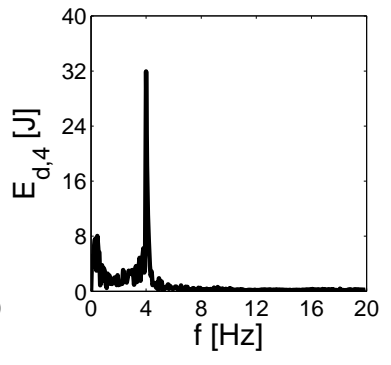

(d)

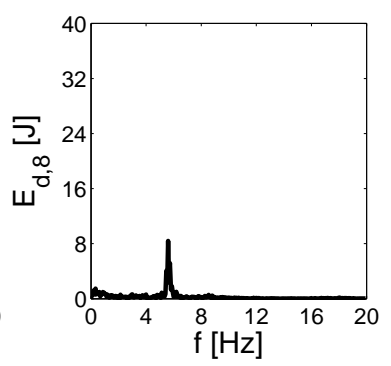

(h)

Figure 12: Modal deformation energy $E_{d, j}(f)$ in the frequency domain for the first eight mode shapes, based on the structural response in the one-way coupling simulation: (a) $\boldsymbol{\Phi}_{1}=(1,3)$, (b) $\boldsymbol{\Phi}_{2}=(1,3)$, (c) $\boldsymbol{\Phi}_{3}=(1,4)$, (d) $\boldsymbol{\Phi}_{4}=(1,4)$, (e) $\boldsymbol{\Phi}_{5}=(1,5)$, (f) $\boldsymbol{\Phi}_{6}=(1,5)$, (g) $\boldsymbol{\Phi}_{7}=(1,5)$, and (h) $\boldsymbol{\Phi}_{8}=(1,5)$.

Although these results are based on one-way coupling simulations of only a single silo, the computationally predicted vibrations correspond well with the observed ovalling vibrations during the 2002 Antwerp storm. While vibration levels are probably still smaller than for the entire silo group arrangement, the simulations already seem to confirm that the mode shapes with the lowest natural frequencies are excited by the aerodynamic pressures.

\subsection{Two-way coupling simulations}

In the two-way coupling simulations, several additional computational issues arise. The first concerns possible interpolation issues at the interface of structural and flow solver. As mentioned, this problem is bypassed by using identical meshes for the wind-structure interface (i.e. the silo surface) in both structural and flow solver. The second concerns the choice and implementation of an implicit coupling scheme to ensure equilibrium at the interface in every time step. In this application, Gauss-Seidel iterations are performed and are found to be stable. Thirdly and finally, the mesh movement of the computational grid in the flow solver is made possible using the arbitrarian Lagrangian-Eulerian (ALE) description as implemented in Ansys Fluent [1]. 
The analysis of the structural response in the two-way coupling simulation is identical to that in the one-way coupling simulation. Based on the structural displacements, the modal deformation energy $E_{\mathrm{d}, j}(t)$ and kinetic energy $E_{\mathrm{k}, j}(t)$ can be determined to investigate the excitation of the different mode shapes in the structural response.

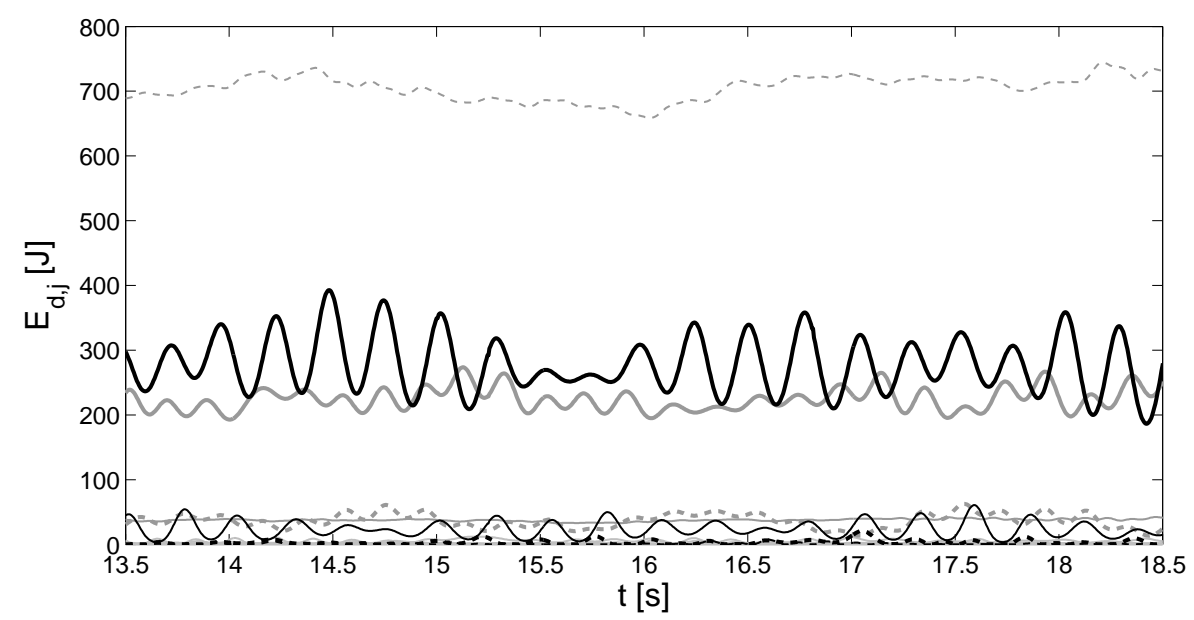

Figure 13: Modal deformation energy $E_{\mathrm{d}, j}$ for the first 20 mode shapes, based on the structural response in the two-way coupling simulation: $\boldsymbol{\Phi}_{1}=(1,3)$ (dashed bold black line), $\boldsymbol{\Phi}_{2}=(1,3)$ (solid bold black line), $\boldsymbol{\Phi}_{3}=(1,4)$ (dashed thin black line), $\boldsymbol{\Phi}_{4}=(1,4)$ (solid thin black line), $\boldsymbol{\Phi}_{5}=(1,5)$ (dashed bold grey line), $\boldsymbol{\Phi}_{6}=(1,5)$ (solid bold grey line), $\boldsymbol{\Phi}_{14}=(2,6)^{*}$ (dashed thin grey line), $\boldsymbol{\Phi}_{18}=(2,6)$ (solid thin grey line), and the remaining mode shapes $\boldsymbol{\Phi}_{j}$ (solid thin light grey lines, with small energy content).

Figure 13 shows the modal deformation energy $E_{\mathrm{d}, j}(t)$ for the first 20 mode shapes, similarly as in figure 11 but now based on the structural response in the two-way coupling simulation. Similarly as for the one-way coupling simulation, mode shape $\Phi_{2}=(1,3)$ has a significant contribution to the deformation energy in the range of approximately $300 \mathrm{~J}$. The 'quasi-static' low-frequency variation of the static component and oscillations at the natural frequency are also observed.

The other mode shapes that were significantly contributing to the structural response in the one-way coupling simulation, namely $\boldsymbol{\Phi}_{1}=(1,3), \boldsymbol{\Phi}_{3}=(1,4)$ and $\boldsymbol{\Phi}_{4}=(1,4)$, now represent only very small to negligible energy variations. Instead, mode shape $\boldsymbol{\Phi}_{6}=(1,5)$ has a significant contribution in the structural displacements, with a large 'quasi-static' part and less pronounced dynamic oscillations. The contribution of mode shape $\boldsymbol{\Phi}_{5}=(1,5)$ is still small in the two-way coupling simulations but has become more important than the contribution of e.g. mode shape $\boldsymbol{\Phi}_{1}=(1,3)$ and $\boldsymbol{\Phi}_{3}=(1,4)$ whose contribution is close to negligible when compared to the one-way coupling results.

A major difference compared to the results of the one-way coupling, is the contribution of mode shape $\boldsymbol{\Phi}_{14}=(2,6)^{*}$ and, although less pronounced, mode shape $\boldsymbol{\Phi}_{18}=(2,6)$ to the deformation energy. The notation $(2,6)^{*}$ is used to characterize the hybrid mode shape combining $(2,6)$ and $(1,2)$. Both mode shapes $\boldsymbol{\Phi}_{14}$ and $\boldsymbol{\Phi}_{18}$ have a mainly 'quasi-static' component and are only little excited dynamically.

Finally, the peak displacements of the structural response in the two-way coupling simulations has doubled compared to the one-way results. The present maximum of $0.06 \mathrm{~m}$ is very high, certainly when considering that the silo is isolated. It is likely but difficult to predict 
how the vicinity of the neighbouring silo structures in the group arrangement will influence the displacement of the silo structure.

From these results, it is clear that the results in the one-way coupling simulations differ from those in the two-way coupling. Both simulations confirm that mode shape $\boldsymbol{\Phi}_{2}=(1,3)$ contributes most to the structural dynamic response, or hence the ovalling vibrations. The secondary mode shapes differ slightly in the one-way and two-way coupling simulations. Nevertheless, the mode shapes with the lowest natural frequencies remain those to be preferentially excited dynamically. An important mismatch between the two coupling approaches is found concerning the 'quasi-static' response of the silo structure: in the two way coupling, two mode shapes with higher natural frequencies are found to contribute significantly to the 'quasi-static' swaying deformation of the silo structure.

These simulations demonstrate the importance of performing fully coupled, two-way simulations for suchlike aeroelastic problems. For the case of the entire 8 by 5 silo group, it is plausible that the two-way coupling simulations will yield even more different results compared to the one-way coupling simulations.

\section{CONCLUSIONS}

To investigate the occurence of wind induced ovalling vibrations in a silo group, the present study proposes a numerical approach. Presently, a single silo in natural wind flow is considered. The silo structure is numerically calculated using a finite element (FE) model and the wind flow around a single silo is investigated using 3D computational fluid dynamics (CFD) simulations. For the validation of the numerical wind flow simulation, a wind tunnel experiment around a rigid structure was set up. Despite several modifications (e.g. scale, inlet conditions, etc.) the experimental results show reasonably good agreement with the numerical results. Because the turbulence model used in the CFD simulations is not adequate for the lower Reynolds number wind flow in the experiments, the separation point is not predicted correctly. Nevertheless good agreement is found for the mean pressures at the windward side of the cylinder surface until separation and also for the fluctuating pressures at the lee side of the cylinder surface. The wind flow pattern is also compared qualitatively to similar cases of finite surface-mounted structures in cross flow. Several similarities can be observed that increase the confidence in the present CFD simulation results.

The coupled wind-structure interaction problem is subsequently simulated using two different coupling approaches. In the one-way coupling simulation, the aerodynamic surface pressures are applied to the FE model of the silo structure as external transient loads without deformation of the flow domain while in the two-way coupling simulation, the interaction of the structural deformation with the wind flow field is taken into account in every time step. Modal decomposition techniques are applied in both simulations to calculate the modal deformation energy for the determination of the excited mode shapes in the structural response. It is found in both coupling approaches that the mode shapes with the lowest natural frequencies are excited dynamically. The results in the one-way coupling simulations differ however from those in the two-way coupling, demonstrating the importance of performing fully coupled simulations for suchlike aeroelastic problems.

\section{REFERENCES}

[1] FLUENT 14.0. User's Guide. Ansys Inc., 2011. 
[2] Scanivalve ZOC 33/64Px Electronic Pressure Scanning Module. Instruction and Service Manual. Scanivalve Corp., July 2010.

[3] Abaqus 6.10. User's Manual. Dassault Systèmes Simulia Corp., 2010.

[4] C.J. Baker. The turbulent horseshoe vortex. Journal of Wind Engineering and Industrial Aerodynamics, 6:9-23, 1980.

[5] BIN. NBN EN 1991-1-4:2005/AC Eurocode 1: Actions on structures - Part 1-4: General actions - Wind actions (+ AC:2010). Belgisch Instituut voor Normalisatie, December 2010.

[6] D. Dooms, G. Degrande, G. De Roeck, and E. Reynders. Finite element modelling of a silo based on experimental modal analysis. Engineering Structures, 28(4):532-542, 2006.

[7] Y. Gai and W.K. Chow. Numerical studies on air flow around a cube. Journal of Wind Engineering and Industrial Aerodynamics, 93:115-135, 2005.

[8] Y. Liu, R.M.C. So, and Z.X. Cui. A finite cantilevered cylinder in a cross-flow. Journal of Fluids and Structures, 20(4):589-609, 2005.

[9] F.R. Menter. Two-Equation Eddy-Viscosity Turbulence Models for Engineering Applications. AIAA Journal, 32(8):1598-1605, 1994.

[10] F.R. Menter, M. Kuntz, and R. Langtry. Ten Years of Industrial Experience with the SST Turbulence Model. In K. Hanjalić, Y. Nagano, and M. Tummers, editors, Proceedings of the Fourth International Symposium on Turbulence, Heat and Mass Transfer, pages 625-632, Antalya, Turkey, October 2003. Begell House, Inc.

[11] M.P. Païdoussis, S.J. Price, and H.C. Suen. Ovalling oscillations of cantilevered and clamped-clamped cylindrical-shells in cross flow: An experimental-study. Journal of Sound and Vibration, 83(4):533-553, 1982.

[12] C.-W. Park and S.-J. Lee. Free end effects on the near wake flow structure behind a finite circular cylinder. Journal of Wind Engineering and Industrial Aerodynamics, 88:231-246, 2000.

[13] C.-W. Park and S.-J. Lee. Effects of free-end corner shape on flow structure around a finite cylinder. Journal of Fluids and Structures, 19:141-158, 2004.

[14] Martinuzzi R. and C. Tropea. The flow around surface-mounted, prismatic obstacles placed in a fully developed channel flow. Journal of Fluids Engineering, Transactions of the ASME, 115:85-92, 1993.

[15] E. Simiu and R. H. Scanlan. Wind Effects on Structures. John Wiley \& Sons, 1986.

[16] A. Smirnov, S. Shi, and I. Celik. Random Flow Generation Technique for Large Eddy Simulations and Particle-Dynamics Modeling. Journal of Fluids Engineering, Transactions of the ASME, 123:359-371, 2001.

[17] V.I. Terekhov, A.I. Gnyrya, and S.V. Korobkov. Vortex pattern of the turbulent flow around a single cube on a flat surface and its heat transfer at different attack angles. Journal of Wind Engineering and Industrial Aerodynamics, 96:1749-1761, 2008. 
[18] Y. Tominaga, A. Mochida, R. Yoshie, H. Kataoka, T. Nozu, M Yoshikawa, and T. Shirisawa. AIJ guidelines for practical applications of CFD to pedestrian wind environment around buildings. Journal of Wind Engineering and Industrial Aerodynamics, 96:17491761, 2008.

[19] M.M. Zdravkovich. Flow Around Circular Cylinders, Volume 1: Fundamentals. Oxford University Press, Oxford, England, 1997. 